\title{
LAS PRIMERAS DAMAS DE LA ORDEN DE MARÍA LUISA
}

\author{
Pere Molas Ribalta \\ Universidad de Barcelona
}

\section{$\underline{\text { RESUMEN }}$}

La Orden de las Damas de la Reina María Luisa fue creada en 1792. En este artículo se estudia las damas de la aristocracia que ingresaron en la misma durante el primer año de la existencia de la orden, las cuales pertenecían diversos grupos de la alta aristocracia y de las élites de gobierno. El análisis de los casos individuales muestra las formas de relación y de sucesión entre las familias de la alta aristocracia, la corte y la alta burocracia.

Palabras clave: aristocracia, España del siglo XVIII.

\section{$\underline{\text { ABSTRACT }}$}

The Orden of Queen María Luisa of Bourbon Parma, wife of king Charles IV was erected in 1792. The paper studies the women of the aristocracy (royal houses excepted) who were admited into the order in the first year. They appartened to families of the high aristocracy and ruling elites. The analysis of individual cases show the forms of relation and succession among families of the high aristocracy, court service and high burocracy.

Keywords: aristocracy, eighteenth century Spain.

La reciente publicación de una obra sobre las damas nobles de la Orden de María Luisa, creada en 1792, nos ofrece una rica información sobre las políticas matrimoniales y sucesorias de las familias de la aristocracia española en la crisis del Antiguo Régimen'. Para las dimensiones de este artículo nos limitamos a las primeras promociones de damas, las que ingresaron en la orden en el año fundacional de 1792. Prescindimos de las que pertenecían a las familias reinantes y nos centramos en las mujeres pertenecientes a la nobleza española para adentrarnos en el conocimiento de las redes de parentesco, estrategias matrimoniales, prácti-

' CEBALlos-ESCALERA y GILA, Alfonso de, Marqués de la FLORESTA, La Real Orden de Damas Nobles de la Reina María Luisa. Real Sociedad Económica Segoviana de Amigos del País. 1998. Citado en adelante como Dumas. 
cas endogámicas, concentración de títulos nobiliarios, procesos de ascensión social y relaciones entre los integrantes de los distintos círculos del poder.

Durante el año 1792 ingresaron en la orden 43 mujeres. Abrían la marcha once damas pertenecientes a distintas ramas de la casa de Borbón o a la de Braganza. E1 21 de abril fueron nombradas 14 damas pertenecientes a familias de la aristocracia y el 17 de diciembre otras 18 . A efectos de análisis comparativo trataremos conjuntamente a estas 32 damas de la alta nobleza que a fines de 1792 constituían, junto a las princesas de sangre real, la orden de damas nobles de María Luisa.

La gran aristocracia estaba experimentando a fines del siglo XVIII un importante proceso de fusión. Una de las damas de la orden nombradas el 17 de diciembre fue la famosa María Josefa Pimentel (1752-1834), condesa de Benavente, duquesa de Gandía, título este último que los Pimentel habían heredado en 1755 después de un duro proceso, tras la extinción de la familia Borja ${ }^{2}$. Doña María Josefa, que había sucedido a su padre en 1763 y había reunido además los importantes títulos de Arcos y Béjar, se había casado en 1771 con Pedro de Alcántara Téllez Girón (1755-1807), que fue el noveno duque de Osuna, tras la muerte de su padre, el octavo duque, Pedro Zoilo Téllez Girón (1728-1787)3.

La condesa de Benavente y duquesa de Osuna tenía como compañeras de la orden a otras mujeres, tanto de su propia familia como de la de su marido. Se trataba de su madre, la condesa viuda de Benavente, María Faustina (1724-1797), que era hija del séptimo duque de Osuna, de la mujer del duque Pedro Zoilo (casados en 1753), María Vicenta Pacheco (1735-1801), hija del duque de Uceda, y de la cuñada de ésta, María de la Portería Fernandez de Velasco (1731-1796), esposa del octavo duque de Uceda, que había sido sucesivamente dama de las reinas Bárbara de Braganza, María Amalia de Sajonia y María Luisa de Parma4.

El matrimonio de Josefa Pimentel y Pedro Téllez Girón tiene su lugar en la Historia social y cultural de fines del Antiguo Régimen. El duque, que antes de la muerte de su padre llevaba el título de marqués de Peñafiel, participó en la con-

\footnotetext{
${ }^{2}$ La posición de los Benavente había sido defendida por el abogado Manuel de Roda, futuro ministro de justicia de Carlos III. Vease el volumen X del Epistolario de Gregorio MAYANS y SISCAR, con estudio introductorio de Antonio MESTRE, Valencia, 1990.

'ALVAREZ de BAENA, José Antonio, Hijos ilistres de Madrid.... Madrid 1798. Edición facsímil Madrid, Editorial Atlas, 1973. IY, p. 268. CEBALLOS-ESCALERA, Afonso, La insigne Orden del Toisón de Oro. (Citada en adelante como Toisón). Madrid, 2000, p. 460, $\mathrm{n}^{\circ}, 776$.

${ }^{4}$ Damas, nos. 14,15 y 28.
} 
quista de Menorca (1782) y siguió una fácil carrera militar que le llevó a ser teniente general (1791) y miembro del Consejo de Guerra. En 1795 entró a formar parte del Consejo de Estado. Obtuvo la gran cruz de la orden de Carlos III (1789) y fue caballero de la del Toisón de Oro (1794) . Participó en la vida política, cultural y económica, en este caso con el proyecto de una gran Compañía de Seguros Marítimos puesta bajo la denominación de la reina ${ }^{\varsigma}$.

La condesa de Benavente y duquesa de Osuna tuvo un papel importante en la Junta de Damas de la Sociedad Económica Matritense de Amigos del País? Vivió hasta 1834 y protagonizó la inflexión liberal de la aristocracia española en los años finales del Antiguo Régimen, junto con sus hijos, por ejemplo, Pedro de Alcántara Téllez Girón Pimentel (nacido en 1786), que llevó el título de príncipe de Anglona ${ }^{8}$. En torno a 1830, el furibundo realista Arias Teijeiro no ahorraba los dicterios a "la vieja de Benavente", a la que llamaba "la satrapona" y a su hijo más bribón y más querido", que era precisamente Anglona?. Una de las hijas de la condesa, Joaquina Téllez Girón y Pimentel (1784-1851), marquesa de Santa Cruz por matrimonio, fue también, en 1830 (en la etapa final del reinado de Fernando VII), dama de la orden de María Luisa. Esta dignidad también la obtuvieron siete de las nietas de doña Josefa, que fueron nombradas en los años posteriores a 1830 y a lo largo del reinado de Isabel II ${ }^{10}$.

Otro grupo familiar era el representado por los duques de Santiesteban, en relación con los de Medinaceli. El segundo duque de Santiesteban, don Antonio de Benavides (1714-1782), había salido de España en 1731 como gentilhombre de cámara del infante don Carlos. Cuando este fue Carlos III, Santiesteban ejerció el cargo de mayordomo mayor del Príncipe de Asturias, alcanzó el grado de teniente general y obtuvo el Toisón de Oro (1764). Se casó en terceras nupcias (1755) con Ana María Fernández de Córdoba y Montcada (1738-1797), hija del

\footnotetext{
${ }^{5}$ ALVAREZ de BAENA, IV, p. 270. BARRIOS, Feliciano, El Consejo de Estado de la Monarquia española (1521-1812). Madrid, 1984, pp. 434-435, 11. 326. OZANAM, Didier. Les diplomates espagnols du XVII siècle. Madrid - Bordeaux, Casa de Velázquez, Maison des Pays Iberiques, 1998, pp. 444-445. Toisón, $n^{\circ} .830$.

"Jovellanos fue muy crítico con esta iniciativa, a la que consideraba un "establecimiento quimérico que camina a la ruina" debido a la "necia ambición del duque de Osuna". Biblioteca de Autores Españoles, tomo 85. Obras Completas. IIl, p. 359.

'DEMERSON, Paula de, "Catálogo de las socias de honor y mérito de la Junta de Damas Matritense (1787-1811)", Anuario del Instituto de Estudios Madrileños (197I), pp. 269-274.

${ }^{8}$ GIRON, Pedro Antonio, Marqués de las AMARILlAS, Memorias. Edición de Ana M". BERAZALUCE. Pamplona, Eunsa, 1979, I, p. 227 nota.

'ARIAS TEIJEIRO, Diarios. Edición de Ana M'. BERAZALUCE. Pamplona, 1967, I, p. 218 nota 269.

In El marqués de Santa Cruz era desde 1829 mayordomo mayor y su esposa fue nombrada en 1830 aya de la princesa Isabel. ARIAS TEIJEIRO, la presentaba como "mujer mala, liberal e inmoral". Diarios, II, p. 322,329 y 336 .
} 
undécimo duque de Medinaceli y de Teresa de Montcada, marquesa de Aitona. Ana María fue una de las damas de María Luisa nombradas en $1792^{11}$.

El título de Santiesteban fue heredado por una hija del segundo matrimonio, Joaquina de Benavides (1746-1805), la cual se casó en 1764 a los 18 años con Luis Fernández de Córdoba (que contaba 15), que fue a su debido tiempo, tras la muerte de su padre (1789), duque de Medinaceli, gran cruz de la orden de Carlos III (1776), caballero del Toisón de Oro (1789), comendador de la de Calatrava, teniente general (1795), caballerizo mayor y mayordomo mayor de la reina ${ }^{12}$.

Una hermana menor, Francisca de Paula Benavides (1763-1829) se había casado en Aranjuez, en 1780, con Diego Antonio Pacheco Téllez Girón y Fernández de Velasco (1754-1811), duque de Frías en 1776 en sucesión de un tío materno y desde 1789 duque de Uceda, en sucesión de su padre, teniente general y consejero de estado en 1802, y embajador de Carlos IV y de José Bonaparte. Francisca de Paula fue otra de las damas de María Luisa. Su hijo Bernardino (1783-1851), duque de Frías, fue político liberal y literato romántico, académico de la Española y presidente del Consejo de Ministros en $1838^{13}$. Otra hermana Benavides, María de la Portería (1748-1798), viuda del marqués de Cullera, fue dama de María Luisa en $1794^{14}$.

También pertenecía a la aristocracia tradicional Felipe López Pacheco, marqués de Villena (1727-1798), caballero del Toisón (1789) gran cruz de la orden de Carlos III, comendador de la Santiago, teniente general, caballerizo y montero mayor. Se habia casado en 1750 con Maria Luisa Centurión, octava marquesa de Estepa (muerta en 1799), que fue otra de las primeras damas de María Luisa ${ }^{15}$.

Maria Isidra de la Cerda Guzmán (1742-1811), condesa de Paredes de Nava y marquesa de la Laguna, fue la heredera de estos títulos de Castilla. Se había casado en 1756 con Diego Ventura de Guzmán Ladrón de Guevara, marqués de Montealegre, conde de Oñate (1738-1805), mayordomo mayor, caballero del Toisón (1780) y gran cruz de la Orden de Carlos III (1776) ${ }^{16}$. Fueron los padres

\footnotetext{
${ }^{11}$ Toisón, $n^{\circ}$. 754. Damas, $n^{\circ}$. 29. FERNAN NUÑEZ, Conde de. Vida de Carlos III. Madrid, Fundación Universitaria Española, 1988. Il, p. 258.

12 Toisón $n^{\circ} .811$. Damas, $\mathrm{n}^{\circ} .30$.

${ }^{13}$ Toison, pp. 474-475, $\mathrm{n}^{\circ}$. 818. Damas. p. 105. OZANAM, pp. 383-384.

${ }^{14}$ Damas, ${ }^{\circ} 50$.

${ }^{15}$ Toisón, $n^{\circ}$. 803. Damas, $n^{\circ} 27$. Vease la dedicatoria a Villena en la Biblioteca de los mejores escritores del reinado de Carlos III, de su protegido Juan Sempere y Guerinos (1788). En realidad había obtenido el título en 1768 por sentencia del Consejo de Castilla en el pleito que siguió con su sobrina Mariana López Pacheco, hija del décimo marqués de Villena. ALVAREZ de BAENA, 1I, pp. 25-26.

'"Damas, n'. 17. Toisón, $\mathrm{n}^{\circ} .780$.
} 
de María Isidra Quintina de Guzmán y la Cerda, la famosa doctora por la universidad de Alcalá y socia de la Económica Matritense ${ }^{17}$.

Otra heredera fue María Teresa Fernández de Córdoba (1736-1792), condesa de Baños y de Leiva, en la Rioja. Se había casado en 1751 con Antonio Joaquín Osorio de Moscoso y Manrique de Zúñiga (1724-1783), hijo del conde de Altamira, caballerizo mayor y mayordomo mayor de Isabel de Farnesio. Con título de conde de Baños, Osorio fue presidente del Consejo de Ordenes desde 1778 hasta su muerte en 1783. Pertenecía a las de Santiago, del Toisón (1764) y de Carlos III y recibió el hábito de la de Santiago cuando fue nombrado para presidir el Consejo de Ordenes ${ }^{18}$. El matrimonio no tuvo sucesión y el condado de Baños fue heredado por Francisca de Sales Portocarrero, la famosa condesa de Montijo, que ingresó en la orden de María Luisa en $1795^{19}$.

El viejo título de conde de Cifuentes había recaído en María Luisa de Silva y González Castejón (1765-1824), cuyo padre, nacido en Viena, en el exilio austracista, culminó su carrera como capitán general borbónico y presidente del Consejo de Castilla en 1791, mientras por parte de madre estaba emparentada con el marqués de Castejón, ministro de marina de Carlos III $^{20}$. Se había casado en 1784 con el aristócrata catalán Juan B. de Queralt y Reart (1758-1803), conde de Santa Coloma y marqués de Besora, familia que con este matrimonio iniciaba su aproximación a los círculos de la corte, que tuvo lugar cuando la familia se trasladó a Madrid tras la muerte del conde ${ }^{21}$.

Otro viejo título castellano, el de Mondéjar, poseído a principios del siglo XVIII por el erudito Gaspar Ibáñez de Segovia, había pasado por vía matrimonial al linaje valenciano de los Bellvís de Moncada, marqueses de Bélgida ${ }^{22}$. Don Pascual Bellvís de Moncada e Ibáñez de Mendoza (Valencia 1727-Madrid 1781), gentilhombre de cámara, gran cruz de la orden de Carlos III (1771), caballero del Toisón (1780), se casó en 1754 con una dama de la corte que concentraba una serie de linajes. Florencia Pizarro (1727-1794), marquesa de San Juan de Piedras Albas, tenía por apellido de su abuela paterna el italiano de Piccolomini de

\footnotetext{
${ }^{17}$ ALVAREZ de BAENA, IV, p. 66. Hay muchas referencias a María Isidra en obras generales sobre la Llustración y las mujeres de la nobleza.

${ }^{18}$ SAENZ BERCEO, Maria del Carmen. El régimen señorial en Castilla: el estado de Baños y Leiva. Logroño. Universidad de la Rioja. 1995. Toisón $\mathrm{n}^{\circ} .759$. Damas, $\mathrm{n}^{2} .18$.

${ }^{19}$ Es ya clásjea la biografia de la condesa por Paula DERMERSON, publicada en 1975 por la Editora Nacional (Madrid).

20 ORTEGA COSTA Y GARCIA OSMA, Presidencia del Conde de Cificentes. Madrid, 1969.

21 Pere MOLAS RIBALTA, "Els cavallers catalans de l'orde de Carles III", Pedralbes. Revista d'Història Maderna, $\pi^{\circ} .16$ (1996), p. 74.

22 Sobre las rentas de esta familia, CATALA SANZ, Jorge A. Rentas y patrimonio de la nobleza valenciana en el siglo XVIII. Madrid, Siglo XXI, 1995, pp. 271 y ss.
} 
Aragona, mientras su madre era doña Juana Josefa de Herrera y Ayala, señora de la isla de Gomera, en las Canarias. Como camarera mayor de la reina, doña Florencia fue la primera dama de la orden de María Luisa que no pertenecía a una familia real ${ }^{23}$.

Su hijo, el marqués de Mondéjar y Bélgida, don Juan de la Cruz Bellvís de Moncada y Pizarro, nacido en 1756, se casó en 1774 con María Alvárez de Toledo (1755-1821), hija del décimo marqués de Villafranca, otra de las damas de la primera promoción de la Orden de María Luisa. El marqués fue gran cruz de la orden de Carlos III (1789). caballerizo mayor de Carlos IV y caballero de la orden del Toisón de Oro (1815) ${ }^{24}$. También perteneció a la primera promoción de damas la hermana de Mondéjar, Maria Concepción (1760-1799), que fue, en 1778, la primera esposa del Vicente Palafox y Centurión, marqués de Ariza, Un tercer hermano, Valentín Bellvís de Moncada y Pizarro (1762-1823), se casó en 1795 con la condesa de Villariezo, que fue dama de María Luisa en 1802 y fueron padres de otra dama de la orden, María Asunción Bellvís de Moncada (1796-1847)25. Aun otra hermana, Maria de las Mercedes (1765-1837), marquesa de Castromonte, ingresó en la orden de María Luisa en $1819^{26}$.

Veamos la situación de las hermanas María Concepción y María Antonia Fernández de Córdoba y Samiento, hijas de la sexta condesa de Salvatierra, María Ana Josefa Sarmiento y de Juan de Mata Fernández de Córdoba, de la casa de Medinaceli, que llevó el título de conde de Salvatierra. La primera se casó en 1771 con Francisco del Castillo Horcasitas (Badalona 1742-Escorial 1798), marqués de Villadarias y conde de Moriana, teniente general, gran cruz de la orden de Carlos III y comendador de la de Santiago ${ }^{27}$. La segunda lo hizo en 1784 con el conde de la Puebla del Maestre (1763-1824), gran cruz de la orden de Carlos III (1791). Tras la guerra de Independencia el conde fue sumiller de corps de Fernando VII y recibió el Toisón de Oro en 1816, mientras su esposa era camarera mayor de la reina Isabel de Braganza ${ }^{28}$.

Es significativo considerar las damas procedentes de la aristocracia aragonesa. En primer lugar las hijas del décimo duque de Hijar, Pedro de Alcántara de Silva Fernández de Híjar (1741-1808), que fue presidente del Consejo de Ordenes y en 1795 consejero de estado. María Pilar (1766-1835) se había casado en 1784

\footnotetext{
${ }^{23}$ Damas, $n^{0}$. 12. El marqués de San Juan de Piedras Albas habia sido consejero de Indias de capa y espada desde 1727 y presidente del mismo organismo de 1763 a 1771.

${ }^{24}$ Damas, $n^{\circ} .20$.

${ }^{25}$ Dumas, nos. 118 y 158.

${ }^{26}$ Damas, $\mathrm{n}^{\circ} 183$.

"Damas ñ. 37. Toisón, n". 832.

${ }^{28}$ Damas, $\mathrm{n}^{\mathrm{n}} .41$. Toisón, $\mathrm{n}^{\circ} .875$.
} 
con su tío abuelo el conde de Aranda ${ }^{29}$, y en segundas nupcias lo hizo en 1802 con otro aristócrata aragonés, Francisco Fernández de Córdoba y Glimes de Brabante (1758-1841), hijo del conde de Sástago, y más adelante primer duque de Alagón en el reinado de Fernando VIl. En el año de su boda Francisco era teniente general. En 1814 recibió la gran cruz de la orden de Carlos III, en 1819 el Toisón de Oro y en 1831 fue ascendido a capitán genera ${ }^{30}$. La hermana de María Pilar, María Teresa (1772-1818), casó en primeras nupcias, en 1790, con Jacobo FitzJames, duque de Berwick (que murió en 1794), y en segundas en $1800 \mathrm{con}$ el marqués de Ariza, Vicente María Palafox y Centurión. El enlace con la casa de Berwick se había completado con el del heredero de Hijar, Agustín (1773-1817), que llevaba el título de duque de Aliaga, gentilhombre de cámara (1789), con María Fernanda Fitz James y Stolberg (1775-1852), otra de las primeras damas de la orden de María Luisa. Aliaga (luego duque de Híjar) fue un aristócrata partícipe de instituciones culturales: director de la Matritense, académico de la Española y de Bellas Artes ${ }^{31}$.

Junto a los duques de Híjar tenemos a las distintas ramas de los Palafox ${ }^{32}$. Entre las primeras damas de la orden de María Luisa encontramos a María Josefa Palafox (1722-1802), hija del segundo marqués de Lazán, casada en 1752 con el tercer duque de Granada de Ega (1713-1769), de la nobleza de Navarra, teniente general $^{33}$. De la casa de los marqueses de Ariza, pertenecieron a la orden dos damas que ya hemos citado, las dos esposas del marqués Vicente Palafox (17561820), que fue gran cruz de la orden de Carlos III (1789) y caballero del Toisón de Oro (1794), mariscal de campo y sumiller de corps. También fue dama de María Luisa una hermana del marqués, Maria Ana Palafox y Silva (1759-1810), casada en 1773 con el navarro Ignacio de Arteaga (1748-1817), marqués de Valmediano y sumiller de corps. caballero del Toisón en $1816^{34}$.

A la nobleza valenciana pertenecía la más anciana de la primera promoción de damas, Laura Castellví, nacida en Viena en 1714, hija de Juan Basilio Castellví, marqués de Villatorcas y de Francisca Mercader, condesa de Cervelló. Se casó en 1754 en la Granja con el castellano de Tierra de Campos Antonio

\footnotetext{
20 OLAECHEA Rafael y FERRER BENIMELI, José Antonio, El Conde de Aranda. Zaragoza, Institución Fernando el Católico, 1998, pp. 191 y ss. "Su segunda esposa".

"Toisón, nº 894. AMARILLAS, Memorias, II, p. 43.

"ALVAREZ de BAENA, IV, p. 372.

32 PLOU GASCON, Miguel. Los Palafox en Aragón. Genealogía y datos biográficios. Zaragoza, Institución Fernando el Católico, 2000.

3. Damas, no. 26. PLOU GASCON, pp. 109-110. Fue la madre de Francisco de Borja Idiáquez y Palafox, cuarto duque de Granada de Ega (Estella 1755 - Madrid 1817), que fue gran cruz de la orden de Carlos III (1790), teniente general (1793), presidente del Consejo de Ordenes y caballero del Toisón de Oro (1916). Toisón, ñ. 877.

${ }^{34}$ Damas n. 26. Toisón $\mathrm{n}^{\circ} 873$.
} 
Osorio de Guzmán (1712), mariscal de campo y caballero de Calatrava (1752). Su hijo, Felipe Carlos, conde de Cervelló, que nació en el Real Sitio de San Ildefonso en 1763, fue caballero de la orden de Alcántara (1770) y de la de Carlos III (1794) y en 1795 ascendido teniente general. La condesa murió en $1797^{35}$.

También era de ascendencia valenciana María Joaquina de los Desamparados de Montserrat y Acuña (1751-1807), hija del marqués de Cruïlles, virrey de Méjico a principios del reinado de Carlos III (1760-1766). Se había casado en 1773 con el viudo Pedro Francisco de Luján, (1727-1794), marqués y luego duque (1780), de Almodóvar del Rio, cortesano, diplomático e intelectual, buen ejemplo del proceso ascensional de un linaje de la alta administración y también de la base social de la Ilustración española: perteneció a las tres grandes academias de la corte y fue el traductor, con seudónimo, de la Historia del abate Raynal $^{36}$.

A la erudición valenciana perteneció el alicantino Antonio de Valcárcel, conde de Lumiares, que fue el segundo esposo de Isabel María Pío de Saboya (1719-1799), marquesa de CastelRodrigo, otra de las damas de $1792^{37}$.

En otro nivel de la nobleza titulada tenemos a la segunda marquesa de Villacastiel, María Teresa Olivares y Cepeda (1748-1800), casada en 1763 con el riojano Manuel Fulgencio Ramírez de Arellano (1731-1797), sexto conde de Murillo, ayudante general de la armada, gentilhombre de cámara y gran cruz de la orden de Carlos III (1791). Su hija Josefa Ramírez de Arellano (1765-1800), condesa de Murillo, casada con el conde de Bornos, ingresó en la orden en $1794^{38}$. A un similar nivel de nobleza pertenecía Francisca de Paula Quincoces (17611808), hija del marqués de Lorenzana, casada con el extremeño José Manuel de Villena, conde de Via Manuel (Badajoz 1752). O Agustina Fermándę de Córdoba, nacida en 1771, hija del marqués del Vado del Maestre, casada a los 17 años con el marqués de Villescas, de edad similar ${ }^{39}$.

A una familia de alta magistratura pertenecía Agustína Adorno (Jerez 1739), hija de un consejero de Castilla, casada en 1758 con Manuel José de Negrete (1736-1818), segundo conde de Campo Alange, regidor de Madrid, que fue teniente general (1784), gran cruz de la orclen de Carlos III (1791), ministro de la

\footnotetext{
"CADENAS VICENT, Vicente. Caballeros de la Orden de Alcóntara... siglo XVIII. Madrid, 1991, II, p. 136.

${ }^{36}$ ALVAREZ de BAENA, IV, p. 226. Toisón, $n^{\circ}$ 807. OZANAM, pp. 323-324.

"Sobre Lumiares, MESTRE, Antonio, Historia, fueros y actitudes politicas, Alicante, 1970, pp. 298 y ss.

${ }^{38}$ Damas, nos. 34 y 56.

"Damas, n'. 31 .
} 
guerra (1790-1795), embajador, consejero de estado (1798), y al fin, ministro y embajador de José I. Su hijo, Francisco Javier Negerte, que fue el cuarto conde de Campo Alange, fue ascendido a teniente general en 1795 a los 32 años, y se casó en 1806 con una dama de María Luisa ${ }^{40}$.

Esposa de un magistrado fue Josefa Joaquina Olazábal, natural de Irún, casada en primeras nupcias con el conde de la Cañada, Juan Acedo Rico, presidente del Consejo de Castilla de 1792 a 1795 y consejero de estado. Tras la muerte de Cañada, su viuda se casó en 1797 con Diego Godoy, el hermano del favorito, que acumulaba cargos militares y dignidades nobiliarias. En el momento de la boda era caballero de la orden de Calatrava (1794) y teniente general (1795). Joaquina Olazábal murió en $1799^{41}$.

Dos de las damas de la primera promoción habían nacido en el extranjero. La aya de las infantas, María Josefa Julia de Chastelet, de origen belga, nacida en 1745, casó a los 14 años con el mariscal de campo conde de Baillancourt, comendador de la orden de Calatrava, que fue gobernador de Alicante desde 1767 hasta su muerte en $1775^{42}$.

También en Bélgica, en la ciudad de Mons, había nacido Carolina de Stolberg (1755-1829), que casó en primeras nupcias con el cuarto duque de Berwick (muerto en 1789), y en segundas con Paolo de Sangro y Merode, príncipe de Castelfranco (Nápoles 1746-Madrid 1815), capitán general (1795), inspector general de caballeria, comendador de la orden de Santiago, gran cruz de la orden de Carlos III (1791), y caballero del Toisón de Oro (1794) ${ }^{43}$.

Entre las damas nombradas el 21 de abril se encontraba una hermana de Godoy, María Antonia, camarista de la reina, casada en 1790 con el aristócrata siciliano Miguel de la Grúa Talamanca, marqués de Branciforte, gran cruz de la orden de Carlos III (1791), que fue virrey de Nueva España y posteriormente fue capitán general (1799) y consejero de estado $(1803)^{44}$. Con este nombramiento se iniciaba la entrada del clan extremeño en la orden. En los años siguientes ingresaron en la misma la madre de Godoy (1794) y su hermana Ramona (1796) y más adelante dos primas políticas, esposas de los generales Alvárez de Faria, parientes del favorito, además de la propia mujer de Godoy, la condesa de Chinchón

\footnotetext{
40 Damas, no. 38, OZANAM, pp. 367-368.

${ }^{41}$ Damas, n". 25.

${ }^{12}$ Damas, na. 22

4.'Damas, nº. 36, p. 104. Toisón, $\mathrm{n}^{\circ} .871$.

${ }^{4}$ NAVARRO GARCIA, Luis y ANTOLIN ESPIGO, María del Pópulo, "El Virrey Marqués de Branciforte", en CALDERON QUIJANO, J. A., Las virreyes de Nueva España en el reinado de Carlos $I V$. Sevilla, Escuela de Estudios Hispano Americanos, 1972. I, pp. 538 y ss.
} 
María Teresa de Borbón y Vallábrgiga y de su hija, Carlota Godoy (1800). El parentesco con el favorito caído no fue obstáculo para que pasados los años, en 1830, ingresara en la orden la hija de Branciforte, Carlota de La Grúa y Godoy, que había nacido en Méjico en 1794 durante el virreinato de su padre y que habia heredado su título (Branciforte había muerto en Marsella en 1812), aunque su madre, la marquesa viuda vivió hasta 1836 y su nombre figuraba en las Guías de forasteros ${ }^{45}$.

Las edades de las damas de la orden se escalonaban desde los 74 años de la condesa de Cervelló hasta los 17 de María Fernanda Fitz-James, la duquesa de Aliaga. De las damas nombradas en abril de 1792 seis tenían más de 50 años, y sólo dos tenían menos de 30 . Entre las mayores se encontraban la marquesa de Castelrodrigo, llamada la princesa Pío (73 años), la condesa viuda de Benavente (68), la marquesa de San Juan de Piedras Alvas (65) y la duquesa de Uceda (61). En cambio la edad de la promoción de diciembre era más joven: las propociones eran de sólo tres damas con más de 50 años (y sólo una de setenta, la duquesa viuda de Granada de Ega), ocho entre 30 y 40 y seis de menos de 30.

La mayor parte de las mujeres se habían casado antes de los 20 años, con escasa diferencia de edad en relación con sus maridos. De todas formas la diferencia de edad se inclinaba casi siempre en favor de los varones que podían casarse con mujeres más jóvenes. El duque de Frías, de 26, casó con Francisca de Paula Benavides, de 17. El segundón Antonio Joaquín de Osorio, de 27, matrimonió con la condesa de Baños, de 15. El conde de Santa Coloma tenía 26 y su mujer, la condesa de Cifuentes, 19. El militar marqués de Villadarias, de 29, casó con una joven mujer de 15 e Ignacio de Arteaga de 25 lo hizo con María Ana Palafox de 14.

En algún caso las mujeres eran mayores que sus esposos. Pedro Zoilo Téllez Girón tenía 25 cuando se casó con María Vicenta Pacheco, de 28. María Josefa Pimentel tenía tres años más que Pedro Téllez Girón. Joaquina de Benavides se casó a los 18 con el heredero de Medinaceli, que tenía 15.

Eran minoría las que se casaron cumplidos los 20. Florencia Pizarro, de 27, lo hizo con el marqués de Bélgida, de la misma edad, los dos viudos. También la princesa Pío y el conde de Lumiares se casaron poco antes de los 30 . El duque de Granada de Ega se caso a los 30 con María Josefa Palafox, hija del marqués de Lazán. La condesa de Cervelló se casó a los 40 años con un hombre de 42.

\footnotetext{
4" Damas, nos. 21 y 268.
} 
Los maridos eran mayores en el caso de segundas nupcias. El conde de Benavente tenía 32 años cuando se casó con la joven María Faustina Téllez Girón, de 14. El duque de Santiesteban tenía 39 años y su tercera esposa, Ana María Fernández de Córdoba, 17. El duque de Almódovar tenía 46 cuando casó con María Joaquina Acuña de 22. Aranda tenía 65 años cuando casó con su sobrina nieta, de 18. Ella volvió a casarse a los 34 con el futuro duque de Alagón que tenía 44.

Mayoritariamente las damas de la orden de María Luisa estaban casadas con hombres que ostentaban dignidades de las órdenes militares, de la de Carlos III o del Toisón de Oro. Veinte de ellos fueron caballeros del Toisón y dicienueve lucían la gran cruz de la orden de Carlos III. Dieciseis por lo menos fueron oficiales generales, la mayor parte tenientes generales e incluso algunos capitanes generales. La mayoría ejercían altos cargos en la corte, lugar donde transcurría su vida. Algunas de estas personas nacieron, se casaron o murieron en los Reales Sitios.

Todas las damas de la orden de María Luisa eran casadas. Algunas debian la dignidad a la posición de sus maridos. Otras a su propia posición en la corte. Veinticuatro de ellas sobrevivieron a sus maridos. De hecho, ocho ya eran viudas en 1792. En cambio sólo cinco muerieron antes que sus maridos

Antes de terminar el año 1792 había muerto una de las damas nombradas el 24 de abril: la condesa de Baños. En los años siguientes la muerte afectó a las damas de mayor edad. La condesa de Cervelló murió a los 83, la princesa Pío a los 80, pero la marquesa de Ariza lo hizo a los 39. También María Josefa Palafox murió relativamente joven, a los 30 años. La mayor parte de damas murieron cumplidos los cincuenta. Josefa Pimentel falleció en 1834, a los 82, La última superviviente de las damas nombradas en 1792 fue la más joven de ella, María Fernanda Fitz-James, que murió en 1852, muy apropiadamente en Versalles, pocos meses antes que Luis Napoleón restaurara el Imperio. 